\title{
Representative Consumer's Risk Aversion and Efficient Risk-Sharing Rules
}

\author{
Chiaki Hara ${ }^{1}$ \\ Institute of Economic Research, Kyoto University \\ James Huang ${ }^{2}$ \\ Department of Accounting and Management, Lancaster University Management School \\ Christoph Kuzmics ${ }^{3,4,5}$ \\ MEDS, Kellogg School of Management, Northwestern University
}

November 6, 2006

\footnotetext{
${ }^{1}$ The corresponding author. Address: Yoshida-Honmachi, Sakyo-ku, Kyoto 606-8501 Japan. Fax: +81 (0)75 753 7148. Email: hara@kier.kyoto-u.ac.jp.

${ }^{2}$ The email address is james.huang@lancaster.ac.uk.

${ }^{3}$ The email address is c-kuzmics@kellogg.northwestern.edu.

${ }^{4}$ We are grateful for their helpful comments to Kazunori Araki, Jeremy Edwards, Günter Franke, Christian Gollier, Piero Gottardi, Jean-Michel Grandmont, Toshiki Honda, Atsushi Kajii, Takashi Kamihigashi, Takashi Kurosaki, Hamish Low, Kazuhiko Ohashi, Heracles Polemarchakis, Makoto Saito, Karl Schmedders, and Jan Werner; and seminar/conference participants at Cambridge, Hitotsubashi, Keio, Kobe, Oita, Osaka, Rhodes Island, Toyama, and Waseda. We would also like to thank the editors and referees for their extremely valuable suggestions on both the contents and exposition of the paper.

${ }^{5}$ The genesis of this paper is as follows. Huang (2000a, 2000b) had first obtained most of the results in this paper. Working independently, Hara and Kuzmics established similar results in a series of manuscripts since December 2001. These outputs are now merged into this paper. Its exposition follows that of Hara and Kuzmics (2005).
} 


\begin{abstract}
We study the representative consumer's risk attitude and efficient risk-sharing rules in a singleperiod, single-good economy in which consumers have homogeneous probabilistic beliefs but heterogeneous risk attitudes. We prove that if all consumers have convex absolute risk tolerance, so must the representative consumer. We also identify a relationship between the curvature of an individual consumer's individual risk sharing rule and his absolute cautiousness, the first derivative of absolute risk-tolerance. Furthermore, we discuss some consequences of these results and refinements of these results for the class of HARA utility functions.
\end{abstract}

JEL Classification Codes: D51, D58, D81, G11, G12, G13.

Keywords: Aggregation, heterogeneous consumers, absolute risk tolerance, mutual fund theorem. 


\section{Introduction}

We consider an exchange economy under uncertainty with a single good and a single consumption period, in which all consumers hold common probability assessments over the state space and yet differing expected utility functions. Two well known properties hold for each Pareto efficient allocation in such an economy. First, every consumer's consumption level is uniquely determined by the aggregate consumption level. Hence every consumer's state-contingent consumption levels can be specified as a function, called the risk sharing rule, of aggregate consumption levels. Second, there exists a representative consumer, having an expected utility function, in the sense that the support price of the single-consumer economy consisting solely of the representative consumer is also the support price for the Pareto efficient allocation of the original, multi-consumer economy.

The benchmark result on this subject matter is the mutual fund theorem. The mutual fund theorem states that if all consumers have a constant, common absolute cautiousness ${ }^{1}$, then the representative consumer also has the same constant absolute cautiousness and all individuals' risk-sharing rules are linear (affine). It has been well perceived in the literature that the assumptions for the mutual fund theorem are so stringent that the applicability of the theorem is questionable. Recent empirical studies into individuals' risk attitudes report a substantial amount of heterogeneity. Barsky et al $(1997)^{2}$ report that $5 \%$ of individuals display an, assumed constant, relative risk aversion of 33 or higher, the median is around 7 , while only $5 \%$ of individuals display a relative risk aversion of 1.3 or lower.

While there have been many contributions dealing with cases in which the assumption of a constant common cautiousness is not met, they tend to concentrate on rather special cases with regards to consumers' risk attitudes, the number of consumers in the economy, wealth distributions across consumers, and probabilistic distributions of initial endowments and asset returns. Moreover, they often appeal to numerical, as opposed to analytical, methods, without fully clarifying the principles behind their results.

In this paper, we obtain qualitative results concerning the implications of heterogeneous absolute cautiousness on the risk-sharing rules and the representative consumer's risk attitude which are true for any Pareto efficient allocation and do not depend on the particular

\footnotetext{
${ }^{1}$ Cautiousness, as defined in Wilson (1968), is the first derivative with respect to consumption of the reciprocal of absolute risk aversion.

${ }^{2}$ We are grateful to an anonymous referee for drawing our attention to this paper.
} 
characteristics of the economy. Should financial markets be complete, equilibrium allocations are Pareto efficient, and our results are therefore true for all equilibrium allocations. The contribution of this paper is, in short, to provide a detailed description of the way in which the representative consumer's absolute cautiousness is not constant and the risk-sharing rules are not linear in general environments.

There are essentially two main results in this paper. The first result is that heterogeneity of individual risk attitudes has a convexifying effect on absolute risk tolerance. For instance, if all individuals have HARA preferences ${ }^{3}$ (and, hence, linear risk-tolerance), but with heterogenous cautiousness, then the representative consumer has strictly convex risk tolerance. In particular this implies that the representative consumer may well be very different from any individual in the economy, his utility function may not even be in the same class as every individual's. While it is impossible to provide closed-form solutions to the representative consumer's utility function we can say a lot about its qualitative properties.

The second result is about the shape of individual consumer's risk sharing rules. While, again, closed-form solutions are not obtainable, we can make very definite statements about the curvature of risk-sharing rules as well as their limiting behavior. We obtain even more concrete results in the case of heterogeneous consumers with HARA preferences.

Throughout the paper we establish our results for the static, one-period model. Hara (2006) showed that it is possible to extend all the results to the multi-period case provided all consumers have time-homogeneous and time-separable expected utility functions and the same time-discount rate. Hence, our results are directly comparable with dynamic models such as those of Mehra and Prescott (1985), Dumas (1989), Campbell and Cochrane (1999), Wang (1996), Benninga and Mayshar (2000), and Chan and Kogan (2002), where there are multiple consumption periods and a common discount rate is assumed.

This paper is organized as follows. Section 2 presents the model and preliminary results. Section 3 provides a formula for the curvature of individual consumers' risk-sharing rules, while Section 4 gives a formula for the representative consumer's absolute risk tolerance. Implications of our results for asset pricing are also given here. Section 6 investigates the limiting behavior of the risk-sharing rules and the representative consumer's risk attitudes when aggregate consumption tends to the upper or lower bounds. Section 7 takes up the special case of HARA preferences and investigates consequences for portfolio insurance. Section 8 concludes.

\footnotetext{
${ }^{3}$ HARA stands for hyperbolic absolute risk aversion.
} 


\section{Model}

There are $I$ consumers, $i \in\{1, \ldots, I\}$. Consumer $i$ has a von-Neumann Morgenstern (also known as Bernoulli) utility function $u_{i}:\left(\underline{d}_{i}, \bar{d}_{i}\right) \rightarrow \mathbb{R}$, where $\underline{d}_{i} \in \mathbb{R} \cup\{-\infty\}, \bar{d}_{i} \in \mathbb{R} \cup\{\infty\}$, and $u_{i}$ is infinitely many times differentiable and satisfies $u_{i}^{\prime}\left(x_{i}\right)>0$ and $u_{i}^{\prime \prime}\left(x_{i}\right)<0$ for every $x_{i} \in\left(\underline{d}_{i}, \bar{d}_{i}\right)$.

The uncertainty of the economy is described by a probability measure space $(\Omega, \mathscr{F}, P)$. The probability measure $P$ specifies the common (objective) belief on the likelihood of the states. Denote by $E$ the expectation with respect to $P$. The aggregate endowment of the economy and each consumer's consumption are both random variables on the probability measure space.

The assumption of a common probabilistic belief and expected utility allows the efficient allocations to be represented in terms of risk-sharing rules. Write $\underline{d}=\sum \underline{d}_{i}$ and $\bar{d}=\sum \bar{d}_{i}$. A risk-sharing rule is an infinitely many times differentiable function $f:(\underline{d}, \bar{d}) \rightarrow\left(\underline{d}_{1}, \bar{d}_{1}\right) \times$ $\cdots \times\left(\underline{d}_{I}, \bar{d}_{I}\right)$ that satisfies $\sum f_{i}(x)=x$ for every $x \in(\underline{d}, \bar{d})$, where $f_{i}$ is the $i$-th coordinate function of $f$.

For each $\lambda=\left(\lambda_{1}, \ldots, \lambda_{I}\right) \in \mathbb{R}_{++}^{I}$ and each $x \in(\underline{d}, \bar{d})$, consider the following maximization problem:

$$
\begin{array}{cl}
\max _{\left(x_{1}, \ldots, x_{I}\right) \in\left(\underline{d}_{1}, \bar{d}_{1}\right) \times \cdots \times\left(\underline{d}_{I}, \bar{d}_{I}\right)} & \sum \lambda_{i} u_{i}\left(x_{i}\right), \\
\text { subject to } & \sum x_{i}=x .
\end{array}
$$

By strict concavity for each $x$, there exists at most one solution to this problem, which we denote by $f_{\lambda}(x)$. If, additionally, the $u_{i}$ satisfy the Inada condition, that is, $u_{i}^{\prime}\left(x_{i}\right) \rightarrow \infty$ as $x_{i} \rightarrow \underline{d}_{i}$ and $u_{i}^{\prime}\left(x_{i}\right) \rightarrow 0$ as $x_{i} \rightarrow \bar{d}_{i}$, then, for every $\lambda$ and $x$, there exists a solution.

Let $\zeta$, measurable in $(\Omega, \mathscr{F}, P)$, denote the aggregate endowment in the economy. It is well known (see e.g. Borch (1962, p. 428) and Wilson (1968), and is nicely explained in Kreps $\left(1990\right.$, Section 5.4)) that a feasible allocation $\left(\zeta_{1}^{*}, \ldots, \zeta_{I}^{*}\right)$ is efficient if and only if it there is a $\lambda \in \mathbb{R}_{++}^{I}$ such that $\zeta_{i}^{*}=f_{\lambda i}(\zeta)$ for every $i .^{4}$

Let $f$ be such an efficient risk-sharing rule. Denote the maximum attained in the problem (1), with the same $\lambda$ as corresponds to $f$, by $u(x)$. We are thereby defining a function $u:(\underline{d}, \bar{d}) \rightarrow \mathbb{R}$, which is the value function of the problem. Since

$$
\sum \lambda_{i} E\left(u_{i}\left(f_{i}(\zeta)\right)\right)=E\left(\sum \lambda_{i} u_{i}\left(f_{i}(\zeta)\right)\right)=E(u(\zeta))
$$

\footnotetext{
${ }^{4}$ To be exact, to establish this equivalence, we need to guarantee that $E\left(u_{i}\left(f_{\lambda i}(\zeta)\right)\right)$ is finite for every $i$. A sufficient condition for this is given in Huang (2002).
} 
the function $u$ can be interpreted as the von-Neumann Morgenstern utility function of the representative consumer corresponding to the efficient risk-sharing rule $f$. Note that the assumption of the common probabilistic belief is crucial for this interpretation of $u$. By the implicit function theorem, $u$ is smooth. To contrast with the representative consumer, we sometimes refer to the $I$ consumers as individual consumers.

The Arrow-Pratt measure of absolute risk aversion of consumer $i$ is defined as

$$
a_{i}\left(x_{i}\right)=-\frac{u_{i}^{\prime \prime}\left(x_{i}\right)}{u_{i}^{\prime}\left(x_{i}\right)}>0 .
$$

The reciprocal of the absolute risk aversion, $1 / a_{i}\left(x_{i}\right)$, is the absolute risk tolerance and denoted by $t_{i}\left(x_{i}\right)$. The Arrow-Pratt measure of relative risk aversion of consumer $i$ is defined, for $x_{i}>0$, as

$$
b_{i}\left(x_{i}\right)=-\frac{u_{i}^{\prime \prime}\left(x_{i}\right) x_{i}}{u_{i}^{\prime}\left(x_{i}\right)}>0 .
$$

The reciprocal of the relative risk aversion, $1 / b_{i}\left(x_{i}\right)$, is the relative risk tolerance and denoted by $s_{i}\left(x_{i}\right)$. All of these are smooth functions.

Wilson (1968, page 129) referred to the first derivative of the absolute risk tolerance, $t_{i}^{\prime}\left(x_{i}\right)$, as cautiousness, but we shall call it the absolute cautiousness, to distinguish it from the relative cautiousness, which is $s_{i}^{\prime}\left(x_{i}\right)$.

The absolute risk aversion $a(x)$, absolute risk tolerance $t(x)$, relative risk aversion $b(x)$, relative risk tolerance $s(x)$, absolute cautiousness $t^{\prime}(x)$, and relative cautiousness $s^{\prime}(x)$ are similarly defined for the representative consumer's utility function $u$. Bear in mind that they depend on the choice of an efficient risk-sharing rule $f$ and hence on the choice of the weights $\lambda$, although none of our analytical results depends on the choice of $\lambda$. In particular, if markets are complete, then the first welfare theorem implies that every equilibrium allocation is efficient. Hence our results are applicable to equilibrium allocations. The values of $\lambda$ are then determined by the individual consumers' initial endowments as well as the choice of an equilibrium in case there is more than one, but our analytical results always hold regardless of the specification of initial endowments or the choice of an equilibrium.

The following lemma is due to Wilson (1968, Theorems 4 and 5).

Lemma 1 (Wilson (1968)) Let $f$ be an efficient risk-sharing rule and $t$ be the representa- 
tive consumer's absolute risk tolerance corresponding to $f$, then, for every $i$ and $x \in(\underline{d}, \bar{d})$,

$$
\begin{aligned}
t(x) & =\frac{1}{f_{i}^{\prime}(x)} t_{i}\left(f_{i}(x)\right), \\
t(x) & =\sum t_{i}\left(f_{i}(x)\right), \\
t^{\prime}(x) & =\sum f_{i}^{\prime}(x) t_{i}^{\prime}\left(f_{i}(x)\right) .
\end{aligned}
$$

Here are some implications of this lemma. First, by $(2), f_{i}^{\prime}(x)>0$, so that $f_{i}$ is strictly increasing for every $x$. This property is called comonotonicity. Also note that $\sum f_{i}^{\prime}(x)=1$ and hence that $f_{i}^{\prime}(x)$ can be interpreted as a probability mass function over the set of individual consumers. Equation (4) then states that the representative consumer's absolute cautiousness is the expected absolute cautiousness of the individual consumers with respect to the this probability mass function. Third, both the absolute risk tolerance and absolute cautiousness are bounded by the individual consumers' counterpart via

$$
\begin{array}{r}
\max \left\{\max _{i} t_{i}\left(f_{i}(x)\right), I \min _{i} t_{i}\left(f_{i}(x)\right)\right\} \leq t(x) \leq I \max _{i} t_{i}\left(f_{i}(x)\right), \\
\min _{i} t_{i}^{\prime}\left(f_{i}(x)\right) \leq t^{\prime}(x) \leq \max _{i} t_{i}^{\prime}\left(f_{i}(x)\right) .
\end{array}
$$

An immediate corollary of inequality (6) is a sufficient condition for the monotonicity of $t$, and hence of $a$.

Corollary 2 If $t_{i}$ (or $a_{i}$ ) is non-decreasing (or non-increasing) for every $i$, then so is $t$ (or a).

\section{Curvature of the Efficient Risk-Sharing Rules}

The following proposition is rich in interpretations.

Proposition 3 For every $i$ and $x \in(\underline{d}, \bar{d})$,

$$
\frac{f_{i}^{\prime \prime}(x)}{f_{i}^{\prime}(x)}=\frac{1}{t(x)}\left(t_{i}^{\prime}\left(f_{i}(x)\right)-t^{\prime}(x)\right) \text {. }
$$

Proof of Proposition 3 By equality (2),

$$
t_{i}\left(f_{i}(x)\right)=t(x) f_{i}^{\prime}(x)
$$

for every $x \in(\underline{d}, \bar{d})$. Differentiating both sides with respect to $x$, we obtain

$$
t_{i}^{\prime}\left(f_{i}(x)\right) f_{i}^{\prime}(x)=t^{\prime}(x) f_{i}^{\prime}(x)+t(x) f_{i}^{\prime \prime}(x)
$$


Rearranging this, we complete the proof.

The intuition behind Proposition 3 is quite simple: Since, by (2), the marginal risksharing $f_{i}^{\prime}(x)$ is proportional to the absolute cautiousness $t_{i}^{\prime}\left(f_{i}(x)\right)$, it must be increased for those consumers for whom the absolute cautiousness increases faster than the average, which is $t^{\prime}(x)$ by $(4)$.

The first implication of is that for every $x \in(\underline{d}, \bar{d})$ and every $i, f_{i}^{\prime \prime}(x)>0$ if $t_{i}^{\prime}\left(f_{i}(x)\right)>$ $t^{\prime}(x) ; f_{i}^{\prime \prime}(x)=0$ if $t_{i}^{\prime}\left(f_{i}(x)\right)=t^{\prime}(x)$; and $f_{i}^{\prime \prime}(x)<0$ if $t_{i}^{\prime}\left(f_{i}(x)\right)<t^{\prime}(x)$. This seems similar to Proposition II of Leland (1980) but in fact differs crucially from it in that the absolute risk tolerance $t$ is derived from the efficient risk-sharing rule $f$ rather than exogenously given. ${ }^{5}$ Its message is otherwise the same: an individual consumer's risk-sharing rule is (locally) convex if he is more absolutely cautious than the representative consumer; (locally) concave if he is less so; and (infinitesimally) linear if they are equally absolutely cautious. In the context of portfolio insurance, as in Leland (1980) and Brennan and Solanki (1981), it implies that only those who are more absolutely cautious than the representative consumer at every level $x$ of aggregate consumption would purchase portfolio insurances. ${ }^{6}$

The second, finer, implication of the proposition is that for every $x \in(\underline{d}, \bar{d})$ and all $i$ and $j$,

$$
t_{i}^{\prime}\left(f_{i}(x)\right) \gtreqless t_{j}^{\prime}\left(f_{j}(x)\right) \text { if and only if } \frac{f_{i}^{\prime \prime}(x)}{f_{i}^{\prime}(x)} \gtreqless \frac{f_{j}^{\prime \prime}(x)}{f_{j}^{\prime}(x)} .
$$

To appreciate this, recall that the ratios of the first and second derivatives, such as $f_{i}^{\prime \prime}(x) / f_{i}^{\prime}(x)$ and $f_{j}^{\prime \prime}(x) / f_{j}^{\prime}(x)$, often appear in expected utility theory. They measure the curvatures of the individual risk-sharing rules $f_{i}$ and $f_{j}$. For example, $f_{i}^{\prime \prime}(x) / f_{i}^{\prime}(x) \geq f_{j}^{\prime \prime}(x) / f_{j}^{\prime}(x)$ for every $x$ if and only if $f_{i}$ is a convex function of $f_{j}$. Proposition 3 therefore implies that the degree of convexity of $f_{i}$ is positively related to consumer $i$ 's absolute cautiousness. That is, the marginal consumption that consumer $i$ receives as the aggregate endowment increases grows at a rate higher than its counterpart for consumer $j$ if consumer $i$ is more absolutely cautious than consumer $j$. What this means in the context of portfolio insurance is that consumer $i$

\footnotetext{
${ }^{5}$ Section 8 contains a more detailed discussion.

${ }^{6}$ Note that Section 14 of Gollier (2001a) investigated a similar maximization problem to (1), albeit in a somewhat different context. Specifically, our set of consumers is replaced by the state space, our utility weights are replaced by the probability measure on the state space, our consumers' utility functions are replaced by state-contingent utility functions, and our resource-feasibility constraint is replaced by the budget constraint. Then Proposition 52 of Gollier (2001a) can be seen to be quite similar to this implication. We are grateful to an anonymous referee for drawing our attention to this analogy.
} 
purchases more portfolio insurance (or options) relative to the size of the reference portfolio he holds than consumer $j$ does.

Our result also shows that the levels of risk tolerance do not matter for the curvatures of the risk-sharing rules, although they do matter for the slopes. ${ }^{7}$

\section{Representative Consumer's Risk Tolerance}

Throughout this section, we let $f$ be an efficient risk-sharing rule and denote by $a, t, b$, and $s$ the representative consumer's absolute risk aversion, absolute risk tolerance, relative risk aversion, and relative risk tolerance, corresponding to $f$.

We show that if every consumer exhibits convex absolute risk-tolerance (non-decreasing absolute cautiousness), then so does the representative consumer. Moreover, even the slightest heterogeneity in consumers' absolute cautiousness would cause the representative consumer's absolute risk-tolerance to be strictly convex (that is, the representative consumer's cautiousness would be strictly increasing). The following formula establishes these conclusions.

Theorem 4 For every $x \in(\underline{d}, \bar{d})$,

$$
t^{\prime \prime}(x)=\sum\left(f_{i}^{\prime}(x)\right)^{2} t_{i}^{\prime \prime}\left(f_{i}(x)\right)+\frac{1}{t(x)} \sum f_{i}^{\prime}(x)\left(t_{i}^{\prime}\left(f_{i}(x)\right)-t^{\prime}(x)\right)^{2} .
$$

Recall that, by equality (4), the mean of the individual consumers' absolute cautiousness $t_{i}^{\prime}\left(f_{i}(x)\right)$ with respect to the probability mass function $f_{i}^{\prime}(x)$ equals the representative consumer's cautiousness $t^{\prime}(x)$. The sum of the second term on the right hand side of $(10)$ is thus the variance of the $t_{i}^{\prime}\left(f_{i}(x)\right)$ with respect to the same probability mass function. It represents the contribution of heterogeneity in absolute cautiousness to the derivative of the representative consumer's absolute cautiousness. As we will see in the subsequent analysis, this theorem has many implications, but its proof is surprisingly simple.

Proof of Theorem 4 Differentiate both sides of equality (4), then we obtain

$$
t^{\prime \prime}(x)=\sum f_{i}^{\prime \prime}(x) t_{i}^{\prime}\left(f_{i}(x)\right)+\sum\left(f_{i}^{\prime}(x)\right)^{2} t_{i}^{\prime \prime}\left(f_{i}(x)\right) .
$$

By $\sum f_{i}^{\prime \prime}(x)=0$ and Proposition 3 ,

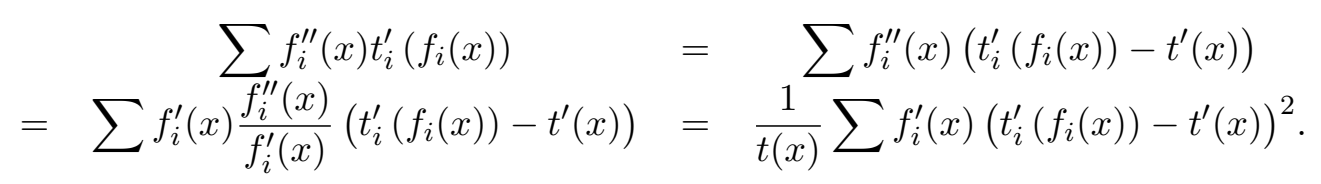

\footnotetext{
${ }^{7}$ We thank Christian Gollier for clarifying this point.
} 
Plug this result into equality (11), then we obtain (10).

The proof makes it clear how the heterogeneity in the individual consumers' absolute cautiousness contributes to the convexity of the representative consumer's absolute cautiousness: By (4), the latter is the weighted average of the former, and, by Proposition 3, the weights $f_{i}^{\prime}(x)$ are increased for those consumers with higher absolute cautiousness. With differing absolute cautiousness, therefore, the representative consumer's counterpart increases, resulting in the convexity of his absolute risk tolerance.

A corollary of this theorem, in terms of the absolute risk tolerance, is:

Corollary 5 If $t_{i}$ is a convex function for every $i$, then so is $t$. If, moreover, the individual consumers' absolute cautiousness are not completely equal at any aggregate consumption level (that is, for every $x \in(\underline{d}, \bar{d})$, there exist two consumers $i$ and $j$ such that $\left.t_{i}^{\prime}\left(f_{i}(x)\right) \neq t_{j}^{\prime}\left(f_{j}(x)\right)\right)$, then $t$ is strictly convex.

Formula (10) suggests that even if all consumers exhibit concave, rather than convex, risk tolerance, the representative consumer may exhibit convex risk tolerance. We can therefore say that the aggregation over heterogeneous consumers tends to induce the representative consumer to exhibit convex risk tolerance.

Calvet, Grandmont, and Lemaire (1999) gave a similar result for the representative consumer's relative risk tolerance. Specifically, denote by $s_{i}\left(x_{i}\right)$ consumer $i$ 's relative risk tolerance $t_{i}\left(x_{i}\right) / x_{i}$ and by $s(x)$ the representative consumer's relative risk tolerance $t(x) / x$. Rewriting their equality (6.10), multiplying $x / s(x)$ to both sides, and rearranging the terms, we obtain the following formula. ${ }^{8}$

Proposition 6 For every $x \in(\underline{d}, \bar{d})$, if $f_{i}(x)>0$ for every $i$, then

$$
s^{\prime}(x)=\sum \frac{f_{i}(x)}{x} f_{i}^{\prime}(x) s_{i}^{\prime}\left(f_{i}(x)\right)+\frac{1}{s(x) x} \sum \frac{f_{i}(x)}{x}\left(s_{i}\left(f_{i}(x)\right)-s(x)\right)^{2} .
$$

It is possible to derive from equality (3) that the mean of the individual consumers' relative risk tolerance $s_{i}\left(f_{i}(x)\right)$ with respect to the probability mass function $f_{i}(x) / x$ equals the representative consumer's relative risk tolerance $s(x)$. The sum in the second term on the right hand side of (10) is thus the variance of the $s_{i}\left(f_{i}(x)\right)$ with respect to this probability mass function. It represents the contribution of heterogeneity in relative risk tolerance to the representative consumer's relative cautiousness $s^{\prime}(x)$.

\footnotetext{
${ }^{8}$ We owe this proof to an anonymous referee
} 
Denote the relative risk aversions by $b_{i}\left(x_{i}\right)=\frac{1}{s_{i}\left(x_{i}\right)}$ and $b(x)=\frac{1}{s(x)}$. A corollary to Proposition 6, which is analogous to Corollary 5 is the following.

Corollary 7 Assume that $\underline{d}_{i} \geq 0$ for every $i$.

1. If $s_{i}$ is a non-decreasing function for every $i$, then so is s. If, moreover, the individual consumers' relative risk tolerances are not completely equal at any aggregate consumption level (that is, for every $x \in(\underline{d}, \bar{d})$, there exist two consumers $i$ and $j$ such that $s_{i}\left(f_{i}(x)\right) \neq$ $\left.s_{j}\left(f_{j}(x)\right)\right)$, then $s$ is strictly increasing.

2. If $b_{i}$ is a non-increasing function for every $i$, then so is $b$. If, moreover, the individual consumers' relative risk aversions are not completely equal at any aggregate consumption level (that is, for every $x \in(\underline{d}, \bar{d})$, there exist two consumers $i$ and $j$ such that $b_{i}\left(f_{i}(x)\right) \neq$ $\left.b_{j}\left(f_{j}(x)\right)\right)$, then $b$ is strictly decreasing.

The symmetry between formulas (10) and (12) is remarkable. The first derivative of the representative consumer's relative risk tolerance and absolute cautiousness are increased by heterogeneity of individual consumers' risk attitudes. Neither of the two formulas is strictly more general than the other, as either accommodates some cases that the other cannot.

\section{Implications of Our Findings}

We now explore how the aggregate endowment (market portfolio) and its options may be mis-priced if a modeler ignores the issues of aggregation and postulates some particular and, given the actual heterogeneous economy, erroneous form for the representative consumer's utility function. Just to simplify the argument, we will assume throughout this section that the individual consumers exhibit constant relative risk aversion, but of differing levels. This implies, in particular, that $(\underline{d}, \bar{d})=\mathbb{R}_{++}$.

Recall that any positive multiple of the representative consumer's marginal utility $u^{\prime}(\zeta)$ is a state price deflator (also known as the state price density and as the pricing kernel). Since $\frac{d}{d x} \ln u^{\prime}(x)=\frac{u^{\prime \prime}(x)}{u^{\prime}(x)}=-\frac{1}{t(x)}$,

$$
u^{\prime}(x)=u^{\prime}(y) \exp \left(-\int_{y}^{x} \frac{1}{t(s)} d s\right)
$$

for any $x>0$ and $y>0$. Thus, if we define, for any fixed $y>0$, a function $\pi: \mathbb{R}_{++} \rightarrow \mathbb{R}_{++}$ 
by

$$
\pi(x)=\frac{\exp \left(-\int_{y}^{x} \frac{1}{t(s)} d s\right)}{E\left(\exp \left(-\int_{y}^{\zeta} \frac{1}{t(s)} d s\right)\right)},
$$

then $\pi(x) / u^{\prime}(x)$ does not depend on $x$ and $E(\pi(\zeta))=1$. Thus $\pi(\zeta)$ has the property of a density function. A derivative asset is a function $\varphi: \mathbb{R}_{++} \rightarrow \mathbb{R}$, which specifies its own payoff as a function of the aggregate endowment. Then the payoff of the derivative asset as a random variable is therefore $\varphi(\zeta)$ and the relative price with respect to the risk-free bond equals $E(\pi(\zeta) \varphi(\zeta))$.

We will consider two types of erroneous postulates a modeler might make for the representative consumer's risk attitudes. First, suppose that the modeler assumes that the representative consumer exhibits linear absolute risk tolerance (hyperbolic absolute risk aversion), such that the absolute risk tolerance and cautiousness are chosen to match the true values at some aggregate consumption level. ${ }^{9}$ Specifically, for any choice of $y>0$, if we define $\widehat{t}: \mathbb{R}_{++} \rightarrow \mathbb{R}$ by $\widehat{t}(x)=t^{\prime}(y)(x-y)+t(y)$, then $\widehat{t}$ is the best linear approximation of $t$ at $y$. Let $\pi$ be as in (13) and define $\widehat{t}: \mathbb{R}_{++} \rightarrow \mathbb{R}_{++}$by

$$
\widehat{\pi}(x)=\frac{\exp \left(-\int_{y}^{x} \frac{d s}{\widehat{t}(s)}\right)}{E\left(\exp \left(-\int_{y}^{\zeta} \frac{d s}{\widehat{t}(s)}\right)\right)} .
$$

This would be the state-price deflator if the representative consumer's risk tolerance were $\widehat{t}$. The following proposition states that this linear approximation $\widehat{\pi}(\zeta)$ underestimates the price of every derivative asset $\varphi(\zeta)$ whenever $\varphi$ is an increasing function.

Proposition 8 Suppose that all individual consumers exhibits constant relative risk aversion and their levels are not completely equal. If a derivative asset $\varphi: \mathbb{R}_{++} \rightarrow \mathbb{R}$ is a non-constant and non-decreasing function, then

$$
E(\pi(\zeta) \varphi(\zeta))>E(\widehat{\pi}(\zeta) \varphi(\zeta))
$$

\footnotetext{
${ }^{9}$ This is equivalent to saying that the absolute risk tolerance of the hypothetical representative consumer is a linear approximation of that of the true representative consumer at some aggregate consumption level. Since the absolute risk tolerance of the (true) representative consumer is a strictly convex function of aggregate consumptions levels and is equal to zero at zero consumption, the absolute risk tolerance of the hypothetical representative consumer is zero at some strictly positive consumption level, and the resulting relative risk aversion is strictly decreasing.
} 
Proof of Proposition 8 By Theorem $4, t^{\prime \prime}(x)>0$ for every $x>0$ and hence $\widehat{t}(x)<t(x)$ for every $x \neq y$. Note that

$$
\frac{\pi(x)}{\widehat{\pi}(x)}=\frac{E\left(\exp \left(-\int_{y}^{\zeta} \frac{d s}{\widehat{t}(s)}\right)\right)}{E\left(\exp \left(-\int_{y}^{\zeta} \frac{d s}{t(s)}\right)\right)} \exp \left(\int_{y}^{x}\left(\frac{1}{\widehat{t}(s)}-\frac{1}{t(s)}\right)\right) .
$$

Thus $\pi(x) / \widehat{\pi}(x)$ is a strictly increasing function of $x$. Hence the distribution of $\zeta$ with respect to the probability measure whose Radon-Nikodym derivative is $\pi(\zeta)$ first-order stochastically dominates the distribution of $\zeta$ with respect to the probability measure whose Radon-Nikodym derivative is $\widehat{\pi}(\zeta)$. Then the strict inequality (15) follows from the assumption that $\varphi$ is nonconstant and non-decreasing.

The above proposition states that the price of any asset with an increasing payoff function (of aggregate endowment) would be under-estimated. Since the aggregate endowment is an increasing function of itself, this implies that the equity premium is under-estimated. Hence a modeler would find it more difficult to match the observed equity premium with reasonable risk preferences if she ignores the convexifying effect of aggregation on the representative consumer's absolute risk tolerance. Note that the proposition above and its proof immediately extend to the more general case of linearly approximating the representative consumer's absolute risk tolerance in any economy in which his utility function exhibits strictly convex absolute risk tolerance.

To consider the second type of an erroneous postulate a modeler might make for the representative consumer's risk attitudes, suppose that a modeler assumes that the representative consumer exhibits constant relative risk aversion. ${ }^{10}$ According to Corollary $7,{ }^{11}$ the representative consumer's relative risk aversion is a strictly decreasing function of aggregate consumption levels, ranging from the maximum to the minimum of the individual consumers' constant relative risk aversion. Thus, if we take, as the hypothetically constant relative risk aversion for the representative consumer, the true relative risk aversion at any consumption level, then neither the actual nor the approximated relative risk aversion would always be higher than the other. Hence it is impossible to obtain a result comparable to Proposition 8.

\footnotetext{
${ }^{10}$ This implies that the absolute risk tolerance of the hypothetical representative consumer is a linear function with the zero intercept on the horizontal axis. When it comes to specifying a utility function, therefore, imposing constant relative risk aversion is a more severe restriction than imposing linear absolute risk tolerance.

${ }^{11}$ And also by Proposition 20, to be presented in Section 7.
} 
Yet, to further explore this question, and its implications for the equity premium puzzle of Mehra and Prescott (1985), note that $\pi(x)=u^{\prime}(x) / E\left(u^{\prime}(\zeta)\right)$. Then the equity premium is $E(\zeta) / E(\pi(\zeta) \zeta)-1=E(\zeta) E\left(u^{\prime}(\zeta)\right) / E\left(\zeta u^{\prime}(\zeta)\right)-1$. According to Gollier (2001a, p. 69), when the variance (and other higher order moments) of $\zeta$ is much smaller than its expected value, the equity premium can be approximated by $\left(\operatorname{Var}(\zeta) /(E(\zeta))^{2}\right) b(E(\zeta))$. Barsky et al (1997, Footnote 18) report that in their experimental study, five percent of subjects display a relative risk aversion of 33 or more; the median relative risk aversion is 7 ; and only five percent display a relative risk aversion of 1.3 or less. Since the representative consumer's relative risk aversion would then range from levels higher than 33 for very low aggregate endowment to levels lower than 1.3 for very high levels of aggregate endowment, in a growing economy, as in the US, we would expect the equity premium to be decreasing over time. This is very much consistent with recent empirical studies by, for example, Blanchard (1993) and Jagannathan et al (2000). If we use the estimate of $\operatorname{Var}(\zeta) /(E(\zeta))^{2}=0.056$ from Gollier (2001a, p. 69), then we obtain an equity premium of roughly $2 \%$ for early years (low aggregate consumption) and one of roughly $0.07 \%$ for later years (with high aggregate consumption). Thus, to argue that the heterogeneity in risk aversion can explain the equity premium, we need to show that the relevant aggregate consumption is so low that the representative consumer is mostly driven by the most risk-averse individuals in the economy. Otherwise, the puzzle may even be further deepened.

Franke, Stapleton, and Subrahmanyam (1999) showed that even if the relative risk aversion is chosen such that the theoretical equity premium is matched to the true equity premium (of the aggregate endowment), the price of any asset with a convex payoff function (of aggregate endowment), such as call and put options, is under-estimated. We should note its consistency with empirical findings: Aït-Sahalia and Lo (2000) derived the representative consumer's relative risk aversion from option prices in a non-parametric, non-linear way. They find that it is decreasing (almost) everywhere. Numerical examples of mis-pricing of options were given in Benninga and Mayshar (2000) and Huang (2003). In particular, Benninga and Mayshar (2000) calculated ratios of the true prices to the predictions based on the assumption of constant relative risk aversion (which is greater than one in most cases) for various exercise prices and various choices of the values of the constant relative risk aversion for the representative consumer. Huang (2003) did a similar exercise for the case where the decreasing relative risk aversion arose from heterogeneous probabilistic beliefs among consumers on the distribution 
of aggregate endowments. Gollier (2005) considered the aggregation problem of heterogeneous probabilistic beliefs when the risk attitudes are also heterogeneous.

While the model of this paper is a static one, the results can be extended to dynamic models, as mentioned in the introduction. The impact of heterogeneity on the risk-free interest rate can then be shown to consist of two factors. First, since the representative consumer displays decreasing relative risk aversion, he tends to become more willing to substitute future consumptions for current consumptions in a growing economy. This causes interest rates to decrease. Second, as discussed in Hara (2006), even if the representative consumer's relative risk aversion is correctly estimated at a given aggregate consumption level, if it is, erroneously, assumed to be constant rather than decreasing, then the representative consumer's relative prudence, which measures the strength of the precautionary saving motive, is underestimated. This leads to an overestimation of the risk-free interest rates. In short, the risk-free interest rates tend to be lower in a heterogenous economy than in a representative-consumer economy.

Note that all our results are true regardless of the particular choice of a fixed vector of utility weights $\lambda_{1}, \ldots, \lambda_{I}$ in the maximization problem (1), and hence, true whether there is wealth inequality among consumers or not. We can also use our findings to study the effect of wealth inequality in the absence of any other form of consumer heterogeneity. To see this point, suppose, as Gollier (2001b) did, that the individual consumers have the same utility function. If all the $\lambda_{i}$ are equal, then $f_{1}(x)=\cdots=f_{I}(x)=x / I$ and, in particular, all the $f_{i}$ are linear. However, if not, then, by Lemma $1, f_{i}(x)>f_{j}(x)$ and $f_{i}^{\prime}(x)>f_{j}^{\prime}(x)$ for all $x$ whenever $\lambda_{i}>\lambda_{j}$, because the $t_{i}$ are all the same and strictly increasing. If the common utility function exhibits linear absolute risk tolerance, then $f_{i}^{\prime \prime}(x) / f_{i}^{\prime}(x)=0$ for all $i$ and $x$, regardless of the values of the $\lambda_{i}$. This is nothing but the mutual fund theorem. ${ }^{12}$ However, if the common utility function exhibits strictly convex absolute risk tolerance, then, by Proposition $3, f_{i}^{\prime \prime}(x) / f_{i}^{\prime}(x)>f_{j}^{\prime \prime}(x) / f_{j}^{\prime}(x)$ for all $x$ whenever $\lambda_{i}>\lambda_{j}$, that is, the wealthier consumers have more convex risk-sharing rules than the poorer ones. If, on the other hand, the common utility function exhibits strictly concave absolute risk tolerance, then this relation is reversed. As for the representative consumer's risk attitudes, we can use Theorem 4 and Proposition 6 to obtain some interesting observations. If all the $\lambda_{i}$ are equal, then $f_{1}(x)=\cdots=f_{I}(x)=x / I$ and hence the second term of the right-hand side of (10) is zero. Thus, whenever the common utility function exhibits concave absolute risk tolerance, so does the representative consumer's utility

\footnotetext{
${ }^{12}$ This theorem will be stated in Section 7 .
} 
function. However, if there is some wealth inequality, then the representative consumer's utility function may exhibit convex absolute risk tolerance over some range of aggregate consumption levels, because the second term is positive. Similarly, Proposition 6 shows that even if the common utility function exhibits increasing relative risk aversion, the representative consumer's utility function may exhibit decreasing relative risk aversion over some range of aggregate consumption levels in the presence of wealth inequality.

\section{Limit Behavior}

In this section, we investigate the limit behavior of the representative consumer's absolute cautiousness, relative risk tolerance (and hence relative risk aversion), and the risk-sharing rules. Roughly speaking, we show that the representative consumer's absolute cautiousness tends to the limit of the most absolutely cautious consumers' counterpart as the aggregate consumption level tends to its upper bound $\bar{d}$ (which may be infinite); and these consumers' share of both the consumption levels, out of the aggregate consumption level, and of marginal consumptions, converges to one. This result is particularly relevant in the analysis of a dynamic growing economy. We also provide an analogous result when the aggregate consumption level tends to its lower bound $\underline{d}$ (which may be negative infinite), but the dominant consumers are then the least absolutely cautious ones. This result is relevant in the analysis of a dynamic contracting economy. ${ }^{13}$ We also make statements of the limit behavior of the representative consumer's relative risk tolerance (and hence relative risk aversion). In the next section, we will apply all of these results to the case where all consumers have HARA preferences.

As a convention of this paper, we allow lim to be $\infty$ or $-\infty$; max and min may be $\infty$ or $-\infty$ accordingly. From the outset, we impose the following assumption.

Assumption 9 For every consumer $i$, both $\lim _{x_{i} \rightarrow \underline{d}_{i}} t_{i}^{\prime}\left(x_{i}\right)$ and $\lim _{x_{i} \rightarrow \bar{d}_{i}} t_{i}^{\prime}\left(x_{i}\right)$ exist.

It is possible to generalize the following propositions by replacing lim by lim sup or lim inf, if the limits do not exist.

\subsection{Absolute Cautiousness and Risk-Sharing Rules}

We first consider the following additional condition. It is intended to cover the case of increasing absolute risk tolerance (and hence decreasing absolute risk aversion).

\footnotetext{
${ }^{13}$ Dumas (1989) and Wang (1996) gave an analysis of this kind in a dynamic economy.
} 
Assumption 10 For every consumer $i, \underline{d}_{i}>-\infty, \bar{d}_{i}=\infty$, and $\lim _{x_{i} \rightarrow \underline{d}_{i}} t_{i}\left(x_{i}\right)=0$.

Define $\bar{I}$ as the set of consumers $i$ such that $\lim _{x_{i} \rightarrow \infty} t_{i}^{\prime}\left(x_{i}\right) \geq \lim _{x_{j} \rightarrow \infty} t_{j}^{\prime}\left(x_{j}\right)$ for every $j$, and $\underline{I}$ as the set of consumers $i$ such that $\lim _{x_{i} \rightarrow \underline{d}_{i}} t_{i}^{\prime}\left(x_{i}\right) \leq \lim _{x_{j} \rightarrow \underline{d}_{j}} t_{j}^{\prime}\left(x_{j}\right)$ for every $j$. The following proposition states that the share of consumers in $\bar{I}$ in the aggregate consumption level, as well as in the marginal consumptions, converges to one as the aggregate consumption level diverges to infinity, and that the representative consumer's absolute cautiousness eventually equals these consumers' absolute cautiousness. It also states that the share of extra consumption in excess of the lower bound which is consumed by consumers in $\underline{I}$ converges to one as the aggregate consumption level converges to the lower bound. Also the representative consumer's absolute cautiousness eventually equals these consumers' absolute cautiousness.

Proposition 11 Under Assumptions 9 and 10,

$$
\begin{aligned}
& \text { 1. } \lim _{x \rightarrow \infty} \sum_{i \in \bar{I}} \frac{f_{i}(x)}{x}=\lim _{x \rightarrow \infty} \sum_{i \in \bar{I}} f_{i}^{\prime}(x)=1 . \\
& \text { 2. } \lim _{x \rightarrow \infty} t^{\prime}(x)=\max _{i \in\{1, \ldots, I\}} \lim _{x_{i} \rightarrow \infty} t_{i}^{\prime}\left(x_{i}\right) . \\
& \text { 3. } \lim _{x \rightarrow \underline{d}} \sum_{i \in \underline{I}} \frac{f_{i}(x)-\underline{d} \underline{d}_{i}}{x-\underline{d}}=\lim _{x \rightarrow \underline{d}} \sum_{i \in \underline{I}} f_{i}^{\prime}(x)=1 . \\
& \text { 4. } \lim _{x \rightarrow \underline{d}} t^{\prime}(x)=\min _{i \in\{1, \ldots, I\}} \lim _{x_{i} \rightarrow \underline{d}_{i}} t_{i}^{\prime}\left(x_{i}\right) .
\end{aligned}
$$

We defer the proof of this proposition to Appendix A, but can now mention its idea in passing: While it is in general difficult to obtain the closed-form solution for each consumer's risksharing rule $f_{i}(x)$, it is much easier to identify the relationship between two consumers' risksharing rules $f_{i}(x)$ and $f_{j}(x)$. If they both have constant (but differing) absolute cautiousness, then one can be written as a power function of the other, with the power equal to the ratio of the two cautiousness. When either of the two is not constant, the equality no longer holds, but some inequality holds for a power function, which is sufficient to establish the limit equalities of Proposition 11.

We next consider the following additional condition. It is intended to cover the case of decreasing absolute risk tolerance (and hence increasing absolute risk aversion), such as quadratic utility functions.

Assumption 12 For every consumer $i, \underline{d}_{i}=-\infty, \bar{d}_{i}<\infty$, and $\lim _{x_{i} \rightarrow \bar{d}_{i}} t_{i}\left(x_{i}\right)=0$. 
Define $\bar{H}$ as the set of consumers $i$ such that $\lim _{x_{i} \rightarrow \bar{d}_{i}} t_{i}^{\prime}\left(x_{i}\right) \geq \lim _{x_{j} \rightarrow \bar{d}_{i}} t_{j}^{\prime}\left(x_{j}\right)$ for every $j$, and $\underline{H}$ as the set of consumers $i$ such that $\lim _{x_{i} \rightarrow-\infty} t_{i}^{\prime}\left(x_{i}\right) \leq \lim _{x_{j} \rightarrow-\infty} t_{j}^{\prime}\left(x_{j}\right)$ for every $j$.

Proposition 13 Under Assumptions 9 and 12,

1. $\lim _{x \rightarrow \bar{d}} \sum_{i \in \bar{H}} \frac{\bar{d}_{i}-f_{i}(x)}{\bar{d}-x}=\sum_{i \in \bar{H}} f_{i}^{\prime}(x)=1$.

2. $\lim _{x \rightarrow \bar{d}} t^{\prime}(x)=\max _{i \in\{1, \ldots, I\}} \lim _{x_{i} \rightarrow \bar{d}_{i}} t_{i}^{\prime}\left(x_{i}\right)$.

3. $\lim _{x \rightarrow-\infty} \sum_{i \in \underline{H}} \frac{f_{i}(x)}{x}=\lim _{x \rightarrow-\infty} \sum_{i \in \underline{H}} f_{i}^{\prime}(x)=1$.

4. $\lim _{x \rightarrow-\infty} t^{\prime}(x)=\min _{i \in\{1, \ldots, I\}} \lim _{x_{i} \rightarrow-\infty} t_{i}^{\prime}\left(x_{i}\right)$.

The proof of this proposition is analogous to that of Proposition 11. We thus omit it.

\subsection{Relative Risk Tolerance and Relative Risk Aversion}

Having done the analysis for the absolute cautiousness, we now move on to the analysis of the relative risk tolerance and relative risk aversion. The key observation for the analysis of the limit behavior of the representative consumer's relative risk tolerance and relative risk aversion is that under suitable assumptions, $\lim _{x_{i} \rightarrow \infty} s_{i}\left(x_{i}\right)=\lim _{x_{i} \rightarrow \infty} t_{i}\left(x_{i}\right) / x_{i}=\lim _{x_{i} \rightarrow \infty} t_{i}^{\prime}\left(x_{i}\right)$ and $\lim _{x_{i} \rightarrow 0} s_{i}\left(x_{i}\right)=\lim _{x_{i} \rightarrow 0} t_{i}\left(x_{i}\right) / x_{i}=\lim _{x_{i} \rightarrow 0} t_{i}^{\prime}\left(x_{i}\right)$ by L'Hôpital's rule. This allows us to apply Proposition 11 to the relative risk aversion. The additional assumption we need for this argument is the following.

Assumption 14 For every consumer $i, \underline{d}_{i}=0$, and $t_{i}$ is a convex function.

This assumption can be satisfied by utility functions exhibiting constant relative risk aversion. Along with other assumptions, it implies that $t_{i}^{\prime}$ is a strictly positive, non-decreasing function.

Thus $t_{i}\left(x_{i}\right) \rightarrow \infty$ as $x_{i} \rightarrow \infty$ and $\lim _{x_{i} \rightarrow \infty} s_{i}\left(x_{i}\right)=\lim _{x_{i} \rightarrow \infty} t_{i}^{\prime}\left(x_{i}\right)$ and $\lim _{x_{i} \rightarrow 0} s_{i}\left(x_{i}\right)=\lim _{x_{i} \rightarrow 0} t_{i}^{\prime}\left(x_{i}\right)$.

The following proposition generalizes Proposition 3 of Benninga and Mayshar (2000).

Proposition 15 Under Assumptions 9, 10, and 14,

1. $\lim _{x \rightarrow \infty} s(x)=\max _{i \in\{1, \ldots, I\}} \lim _{x_{i} \rightarrow \infty} s_{i}\left(x_{i}\right)$ and $\lim _{x \rightarrow 0} s(x)=\min _{i \in\{1, \ldots, I\}} \lim _{x_{i} \rightarrow 0} s_{i}\left(x_{i}\right)$.

2. $\lim _{x \rightarrow \infty} b(x)=\min _{i \in\{1, \ldots, I\}} \lim _{x_{i} \rightarrow \infty} b_{i}\left(x_{i}\right)$ and $\lim _{x \rightarrow 0} b(x)=\max _{i \in\{1, \ldots, I\}} \lim _{x_{i} \rightarrow 0} b_{i}\left(x_{i}\right)$. 
Proof of Proposition 15 1. By Proposition 11, $\lim _{x \rightarrow \infty} t^{\prime}(x)$ exists and, by L'Hôpital's rule, equals $\lim _{x \rightarrow \infty} s(x)$. By the same proposition, $\lim _{x \rightarrow \infty} t^{\prime}(x)$ equals $\lim _{x_{i} \rightarrow \infty} t_{i}^{\prime}\left(x_{i}\right)$ for every $i \in \bar{I}$, which equals $\max _{i} \lim _{x_{i} \rightarrow \infty} s_{i}\left(x_{i}\right)$. Hence $\lim _{x \rightarrow \infty} s(x)=\max _{i \in\{1, \ldots, I\}} \lim _{x_{i} \rightarrow \infty} s_{i}\left(x_{i}\right)$.

As for the limit as $x \rightarrow 0$, note that as $x \rightarrow 0, f_{i}(x) \rightarrow 0$ and hence $t_{i}\left(f_{i}(x)\right) \rightarrow 0$. Thus $t(x)=\sum t_{i}\left(f_{i}(x)\right) \rightarrow 0$. This shows that L'Hôpital's rule is applicable and the rest of the argument is as before.

2. This follows from part 1 and the definition of $b$ and $s$.

Now define $\bar{J}$ as the set of consumers $i$ such that $\lim _{x_{i} \rightarrow \infty} s_{i}\left(x_{i}\right) \geq \lim _{x_{j} \rightarrow \infty} s_{j}\left(x_{j}\right)$ for every $j$, which is equivalent to $\lim _{x_{i} \rightarrow \infty} b_{i}\left(x_{i}\right) \leq \lim _{x_{j} \rightarrow \infty} b_{j}\left(x_{j}\right)$ for every $j$. Analogously, define $\underline{J}$ as the set of consumers $i$ such that $\lim _{x_{i} \rightarrow 0} s_{i}\left(x_{i}\right) \leq \lim _{x_{j} \rightarrow 0} s_{j}\left(x_{j}\right)$ for every $j$, which is equivalent to $\lim _{x_{i} \rightarrow 0} b_{i}\left(x_{i}\right) \geq \lim _{x_{j} \rightarrow 0} b_{j}\left(x_{j}\right)$ for every $j$. We have already seen that $\bar{J}=\bar{I}$ and $\underline{J}=\underline{I}$ under Assumption 14. Proposition 11 thus implies the following:

Proposition 16 Under Assumptions 9, 10, and 14,

1. $\lim _{x \rightarrow \infty} \sum_{i \in \bar{J}} \frac{f_{i}(x)}{x}=\lim _{x \rightarrow \infty} \sum_{i \in \bar{J}} f_{i}^{\prime}(x)=1$.

2. $\lim _{x \rightarrow 0} \sum_{i \in \underline{J}} \frac{f_{i}(x)}{x}=\lim _{x \rightarrow 0} \sum_{i \in \underline{J}} f_{i}^{\prime}(x)=1$.

\section{Linear Absolute Risk Tolerance}

Combining the preceding results and assuming that all consumers' utility functions exhibit linear absolute risk tolerance, we show in this section that an individual consumer's risksharing rule is either everywhere concave, everywhere convex, or has a unique inflection point below which it is convex and above which it is concave.

Mathematically, a utility function $u_{i}:\left(\underline{d}_{i}, \bar{d}_{i}\right) \rightarrow \mathbb{R}$ exhibits linear absolute risk tolerance if, for the corresponding absolute risk tolerance $t_{i}$, there exist two numbers $\tau_{i}$ and $\gamma_{i}$ such that

$$
t_{i}\left(x_{i}\right)=\tau_{i}+\gamma_{i} x_{i}
$$

for every $x_{i} \in\left(\underline{d}_{i}, \bar{d}_{i}\right)$. This is equivalent to hyperbolic absolute risk aversion $a_{i}\left(x_{i}\right)=\frac{1}{\tau_{i}+\gamma_{i} x_{i}}$ and constant absolute cautiousness $t_{i}^{\prime}\left(x_{i}\right)=\gamma_{i}$.

Note that the right hand side of equality (17) is of course positive for every $x_{i} \in\left(\underline{d}_{i}, \bar{d}_{i}\right)$ but $\tau_{i}$ and $\gamma_{i}$ may be positive, zero, or negative. However, if $\gamma_{i}=0$, then $\tau_{i}>0$ and we take 
$\underline{d}_{i}=-\infty$ and $\bar{d}_{i}=\infty$. On the other hand, if $\gamma_{i}>0$ then we take $\underline{d}_{i}=-\tau_{i} / \gamma_{i}$ and $\bar{d}_{i}=\infty$ and hence $t_{i}\left(x_{i}\right)=\gamma_{i}\left(x_{i}-\underline{d}_{i}\right)$ and $t_{i}\left(x_{i}\right) \rightarrow 0$ as $x_{i} \rightarrow \underline{d}_{i}$. If $\gamma_{i}<0$, then $\underline{d}_{i}=-\infty$ and $\bar{d}_{i}=-\tau_{i} / \gamma_{i}$ and hence $t_{i}\left(x_{i}\right)=-\gamma_{i}\left(\bar{d}_{i}-x_{i}\right)$ and $t_{i}\left(x_{i}\right) \rightarrow 0$ as $x_{i} \rightarrow \bar{d}_{i}$. Indeed, although we do not provide the proof here, these choices of $\underline{d}_{i}$ and $\bar{d}_{i}$ are the only ones that allows $u_{i}$ to satisfy the Inada condition.

As in the previous sections, let $f:(\underline{d}, \bar{d}) \rightarrow\left(\underline{d}_{1}, \bar{d}_{1}\right) \times \cdots \times\left(\underline{d}_{I}, \bar{d}_{I}\right)$ be an efficient risksharing rule, and denote the representative consumer's absolute risk aversion, absolute risk tolerance, and relative risk aversion by $a, t$, and $b$, all corresponding to $f$.

As documented in, for example, Wilson (1968), Huang and Litzenberger (1988, Sections 5.15 and 5.26), Magill and Quinzii (1996, Proposition 16.3), Gollier (2001a, Section 21.3.3), and LeRoy and Werner (2001, Section 15.6)), the celebrated mutual fund theorem says, in our notation, that if $\gamma_{1}=\cdots=\gamma_{I}$, then $f_{i}$ is affine for every $i$ and $t$ is affine as well ${ }^{14}$.

Denote $\bar{\gamma}=\max \left\{\gamma_{1}, \ldots, \gamma_{I}\right\}$ and $\underline{\gamma}=\min \left\{\gamma_{1}, \ldots, \gamma_{I}\right\}$. Then, according to the notation in the previous section, $\bar{I}=\left\{i \mid \gamma_{i}=\bar{\gamma}\right\}$ and $\underline{I}=\left\{i \mid \gamma_{i}=\underline{\gamma}\right\}$. Then $\bar{I}$ is the set of the most absolutely cautious consumers and $\underline{I}$ is the set of the least absolutely cautious consumers. All consumers are equally cautious if and only if $\bar{\gamma}=\underline{\gamma}$. Of course, this case has been dealt with by the mutual fund theorem, and we thus assume in the remainder of this section that $\bar{\gamma}>\underline{\gamma}$.

The first result of this section is concerned with the representative consumer's absolute risk tolerance.

Proposition 17 Assume that $\bar{\gamma}>\underline{\gamma}$. Then $t^{\prime \prime}(x)>0$ for every $x \in(\underline{d}, \bar{d}), \lim _{x \rightarrow \bar{d}} t^{\prime}(x)=\bar{\gamma}$, and $\lim _{x \rightarrow \underline{d}} t^{\prime}(x)=\underline{\gamma}$

Proof of Proposition 17 The first part of this proposition follows from Corollary 5. The second and third parts follow from Propositions 11 and 13, respectively. ${ }^{15}$

The main result of this section is the following classification of risk-sharing rules.

Theorem 18 Assume that $\bar{\gamma}>\underline{\gamma}$.

\footnotetext{
${ }^{14}$ We should also add that Kurosaki (2001) claimed that if all consumers exhibit constant relative risk aversion, then the logarithmic risk-sharing rule, which assigns the mean of the logs of the consumers' consumption levels to the log of each individual consumer's consumption level, is linear with a slope proportional to his own relative risk tolerance.

${ }^{15}$ Strictly speaking Propositions 11 and 13 were shown to hold only under the assumption that the consumers' levels of cautiousness are either all strictly negative or strictly positive. Proposition 17 is still true even without these assumptions.
} 
1. $f_{i}^{\prime \prime}(x)>0$ for every $i \in \bar{I}$ and $x \in(\underline{d}, \bar{d})$.

2. $f_{i}^{\prime \prime}(x)<0$ for every $i \in \underline{I}$ and $x \in(\underline{d}, \bar{d})$.

3. For every $i \notin \bar{I} \cup \underline{I}$, there exists a unique $y_{i} \in\left(\underline{d}_{i}, \bar{d}_{i}\right)$ such that $f_{i}^{\prime \prime}(x)>0$ for every $x<y_{i}$ and $f_{i}^{\prime \prime}(x)<0$ for every $x>y_{i}$.

4. For the $y_{i}$ defined as in part $3, y_{i}<y_{j}$ if $\gamma_{i}<\gamma_{j} ; y_{i}=y_{j}$ if $\gamma_{i}=\gamma_{j}$; and $y_{i}>y_{j}$ if $\gamma_{i}>\gamma_{j}$.

Proof of Theorem 18 By Proposition 17, $\underline{\gamma}<t^{\prime}(x)<\bar{\gamma}$ for every $x \in(\underline{d}, \bar{d})$. Parts 1 and 2 then follow from Proposition 3. As for part 3, note that Proposition 17 implies that $t^{\prime}:(\underline{d}, \bar{d}) \rightarrow(\underline{\gamma}, \bar{\gamma})$ is strictly increasing and onto. Hence, for every $i \notin \bar{I} \cup \underline{I}$, there exists a unique $y_{i} \in\left(\underline{d}_{i}, \bar{d}_{i}\right)$ such that $\gamma_{i}=t^{\prime}\left(y_{i}\right)$. Since $\gamma_{i}=t_{i}^{\prime}\left(f_{i}(x)\right)$ for every $x$, Proposition 3 implies that $y_{i}$ has the property of part 3. Part 4 also follows from this property of $y_{i}$ and the fact that $t^{\prime}$ is strictly increasing.

Parts 1 and 2 of Theorem 18 have been obtained by Leland (1980) and Brennan and Solanki (1981), who considered the expected utility maximization problem of a consumer who chooses over state-contingent claims of a reference portfolio. Holding the underlying asset and a put option is equivalent to holding cash and a call option of the same exercise price, but these are also equivalent to having a portfolio insurance as well. In all of these cases, the generated return is a convex function of the values of the portfolio. They were thus led to identify conditions on the consumer's utility function for his optimal choice of return to be a convex function of the value of the portfolio.

The most important differences between this work and theirs is that they took the representative consumer's risk aversion as given, while we derive it as a result of efficient risk-sharing among heterogeneous consumers. In fact, our result shows that the case Leland (1980) analyzed on page 589, where the individual and the representative consumers exhibit constant but differing relative risk aversion, is in fact impossible, if all the other consumers also exhibit constant relative risk aversion.

Also, the importance of part 3 of Theorem 18, i.e. the fact that risk-sharing rules for intermediate linearly risk tolerant consumers are initially convex and eventually concave, cannot be overemphasized. It is exactly the point that is not present in the analysis of Leland (1980) and Brennan and Solanki (1981). When individual consumers have differing degrees 
of absolute cautiousness, the representative consumer's absolute cautiousness is strictly increasing, ranging from the smallest to the largest. If an individual consumer has neither the smallest nor the largest absolute cautiousness, then his absolute cautiousness must be caught up with by the representative consumer's counterpart at some aggregate consumption level. Below this level, his risk-sharing rule is convex, and, above this level, it is concave. Then only consumers with the smallest relative risk aversion (the largest absolute cautiousness) would buy portfolio insurance, as the others' risk-sharing rules would eventually become concave. This significantly undermines the applicability of the results of Leland (1980) and Brennan and Solanki (1981). They are valid in a two-consumer economy, but do not generalize to an economy with a large number of consumers with diverse levels of relative risk aversion. This confirms a conjecture by Dumas (1989), who concentrated on a two-consumer economy but concluded by suggesting that the equilibrium behavior of a three-consumer economy may be critically different from that in his two-consumer economy.

Part 3 of Theorem 18 can be partially extended to the general case. Call an intermediate consumer a consumer whose absolute cautiousness is neither the largest nor the smallest when aggregate endowment tends to either of its limits. Then this intermediate consumer's risksharing rule must be initially convex and eventually concave. Given smoothness of all utility functions, this consumer's risk-sharing rule must have at least one inflection point.

The next proposition is concerned with the total proportion of consumption levels consumed by those consumers with the largest or smallest absolute cautiousness. It immediately follows from Propositions 11 and 13. We thus omit the proof.

\section{Proposition 19}

1. If $\underline{\gamma}>0$, then $\lim _{x \rightarrow \infty} \sum_{i \in \bar{I}} \frac{f_{i}(x)}{x}=1$ and $\lim _{x \rightarrow \underline{d}} \sum_{i \in \underline{I}} \frac{f_{i}(x)-\underline{d}_{i}}{x-\underline{d}}=1$.

2. If $\bar{\gamma}<0$, then $\lim _{x \rightarrow-\infty} \sum_{i \in \underline{I}} \frac{f_{i}(x)}{x}=1$ and $\lim _{x \rightarrow \bar{d}} \sum_{i \in \bar{I}} \frac{\bar{d}_{i}-f_{i}(x)}{\bar{d}-x}=1$.

If we further assume that $\underline{d}_{i}=0, \tau_{i}=0$, and $\gamma_{i}>0$ for every $i$, then $b_{i}\left(x_{i}\right)=1 / \gamma_{i}$ and hence $u_{i}$ exhibits constant relative risk aversion $1 / \gamma_{i}$. The following result, which follows from Proposition 17, is concerned with this case.

Proposition 20 Assume that $\underline{d}_{i}=0, \tau_{i}=0$, and $\gamma_{i}>0$ for every $i$, and that $\bar{\gamma}>\underline{\gamma}$.

1. $\lim _{x \rightarrow \infty} s(x)=\bar{\gamma}$ and $\lim _{x \rightarrow 0} s(x)=\underline{\gamma}$. 
2. $\lim _{x \rightarrow \infty} b(x)=1 / \bar{\gamma}$ and $\lim _{x \rightarrow 0} b(x)=1 / \underline{\gamma}$.

\section{Conclusion}

We have presented detailed properties of the efficient risk-sharing rules and the representative consumer's risk attitude in an economy under uncertainty where individual consumers have homogeneous probabilistic beliefs over the state space but heterogeneous risk attitudes. In particular, we have shown that heterogeneity in the consumers' absolute cautiousness, which is the derivative of the reciprocal of the Arrow-Pratt measure of absolute risk aversion, is a key factor for the curvature of the risk-sharing rules. We have also shown that the heterogeneity in the individual consumers' risk attitudes tends to make the representative consumer's absolute risk tolerance convex and relative risk aversion decreasing. We have explored the implications of these findings for asset pricing and portfolio insurance. In particular, we have identified the source of underestimation of the equity premium when the representative consumer is erroneously assumed to exhibit certain types of risk attitudes, and the nature of the optimal portfolio insurance for consumers who are neither the most nor least risk-averse in the economy.

\section{A Proof of Proposition 11}

To prove Proposition 11, we need two lemmas. The first one is concerned with the limit behavior of the ratio of two individual consumers' risk-sharing rules and their derivatives when the aggregate consumption levels diverge to infinity.

Lemma 21 Under Assumptions 9 and 10, if $\lim _{x_{i} \rightarrow \infty} t_{i}^{\prime}\left(x_{i}\right)<\lim _{x_{j} \rightarrow \infty} t_{j}^{\prime}\left(x_{j}\right)$, then $\lim _{x \rightarrow \infty} \frac{f_{i}(x)}{f_{j}(x)}=$ $\lim _{x \rightarrow \infty} \frac{f_{i}^{\prime}(x)}{f_{j}^{\prime}(x)}=0$.

Proof of Lemma 21 Let two real numbers $\delta_{i}$ and $\delta_{j}$ be such that $\lim _{x_{i} \rightarrow \infty} t_{i}^{\prime}\left(x_{i}\right)<\delta_{i}<\delta_{j}<$ $\lim _{x_{j} \rightarrow \infty} t_{j}^{\prime}\left(x_{j}\right)$. Since $\bar{d}_{i}=\infty$ and $t_{i}\left(x_{i}\right)>0$ for every $x_{i}, \lim _{x_{i} \rightarrow \infty} t_{i}^{\prime}\left(x_{i}\right) \geq 0$. Hence $\delta_{i}>0$ and $\delta_{j}>0$. Then let $\underline{x}>\underline{d}$ be such that $t_{i}^{\prime}\left(x_{i}\right)<\delta_{i}<\delta_{j}<t_{j}^{\prime}\left(x_{j}\right)$ for every $x_{i} \geq f_{i}(\underline{x})$ and $x_{j} \geq f_{j}(\underline{x})$. Then, for such $x_{i}$ and $x_{j}$,

$$
\begin{gathered}
t_{i}\left(x_{i}\right)<\delta_{i}\left(x_{i}-f_{i}(\underline{x})\right)+t_{i}\left(f_{i}(x)\right), \\
t_{j}\left(x_{j}\right)>\delta_{j}\left(x_{j}-f_{j}(\underline{x})\right)+t_{j}\left(f_{j}(x)\right) .
\end{gathered}
$$


By equality $(2)$ and the fact that a consumer's absolute risk aversion $a_{i}(\cdot)$ is the reciprocal of his absolute risk tolerance $t_{i}(\cdot)$,

$$
\int_{\underline{x}}^{x} a_{i}\left(f_{i}(z)\right) f_{i}^{\prime}(z) d z=\int_{\underline{x}}^{x} a_{j}\left(f_{j}(z)\right) f_{j}^{\prime}(z) d z
$$

for every $x \geq \underline{x}$. By integration by parts, this is equivalent to

$$
\int_{f_{i}(\underline{x})}^{f_{i}(x)} a_{i}(z) d z=\int_{f_{j}(\underline{x})}^{f_{j}(x)} a_{j}(z) d z .
$$

Thus

$$
\int_{f_{i}(\underline{x})}^{f_{i}(x)} \frac{d z}{\delta_{i}\left(z-f_{i}(\underline{x})\right)+t_{i}\left(f_{i}(\underline{x})\right)}<\int_{f_{j}(\underline{x})}^{f_{j}(x)} \frac{d z}{\delta_{j}\left(z-f_{j}(\underline{x})\right)+t_{j}\left(f_{j}(\underline{x})\right)} .
$$

Take the integral and then the exponential of both sides, then we obtain

$$
\left(\frac{\delta_{i}\left(f_{i}(x)-f_{i}(\underline{x})\right)+t_{i}\left(f_{i}(\underline{x})\right)}{t_{i}\left(f_{i}(\underline{x})\right)}\right)^{1 / \delta_{i}}<\left(\frac{\delta_{j}\left(f_{j}(x)-f_{j}(\underline{x})\right)+t_{j}\left(f_{j}(\underline{x})\right)}{t_{j}\left(f_{j}(\underline{x})\right)}\right)^{1 / \delta_{j}},
$$

because $0<\delta_{i}<\delta_{j}$. Thus

$$
f_{i}(x)-f_{i}(\underline{x})+\frac{t_{i}\left(f_{i}(\underline{x})\right)}{\delta_{i}}<k\left(f_{j}(x)-f_{j}(\underline{x})+\frac{t_{j}\left(f_{j}(\underline{x})\right)}{\delta_{j}}\right)^{\delta_{i} / \delta_{j}},
$$

where

$$
k=\frac{t_{i}\left(f_{i}(\underline{x})\right)}{\delta_{i}}\left(\frac{\delta_{j}}{t_{j}\left(f_{j}(\underline{x})\right)}\right)^{\delta_{i} / \delta_{j}}>0 .
$$

Since $0<\delta_{i} / \delta_{j}<1$,

$$
\frac{f_{i}(x)-f_{i}(\underline{x})+\frac{t_{i}\left(f_{i}(\underline{x})\right)}{\delta_{i}}}{f_{j}(x)-f_{j}(\underline{x})+\frac{t_{j}\left(f_{j}(\underline{x})\right)}{\delta_{j}}} \rightarrow 0
$$

as $x \rightarrow \infty$. Hence $f_{i}(x) / f_{j}(x) \rightarrow 0$ as $x \rightarrow \infty$.

By equality (1),

$$
\frac{f_{i}^{\prime}(x)}{f_{j}^{\prime}(x)}=\frac{t_{i}\left(f_{i}(x)\right)}{t_{j}\left(f_{j}(x)\right)}<\frac{\delta_{i}}{\delta_{j}} \frac{f_{i}(x)-f_{i}(\underline{x})+\frac{t_{i}\left(f_{i}(\underline{x})\right)}{\delta_{i}}}{f_{j}(x)-f_{j}(\underline{x})+\frac{t_{j}\left(f_{j}(\underline{x})\right)}{\delta_{j}}} .
$$

By (19), the far right hand side converges to 0 . Hence $f_{i}^{\prime}(x) / f_{j}^{\prime}(x) \rightarrow 0$.

The next lemma is concerned with the limit behavior of the risk-sharing rules when the aggregate consumption levels converge to the lower bound.

Lemma 22 Under Assumptions 9 and 10, if $\lim _{x_{j} \rightarrow \underline{d}_{j}} t_{i}^{\prime}\left(x_{j}\right)<\lim _{x_{i} \rightarrow \underline{d}_{i}} t_{i}^{\prime}\left(x_{i}\right)$, then $\lim _{x \rightarrow \underline{d}} \frac{f_{i}(x)-\underline{d}_{i}}{f_{j}(x)-\underline{d}_{j}}=$ $\lim _{x \rightarrow \underline{d}} \frac{f_{i}^{\prime}(x)}{f_{j}^{\prime}(x)}=0$. 
Proof of Lemma 22 Let two real numbers $\delta_{i}$ and $\delta_{j}$ be such that $\lim _{x_{j} \rightarrow \underline{d}_{j}} t_{j}^{\prime}\left(x_{j}\right)<\delta_{j}<\delta_{i}<$ $\lim _{x_{i} \rightarrow \underline{d}_{i}} t_{i}^{\prime}\left(x_{i}\right)$. Since $t_{j}\left(x_{j}\right) \geq 0$ for every $x_{j}$ and $t_{j}\left(x_{j}\right) \rightarrow 0$ as $x_{j} \rightarrow \underline{d}_{j}$, we have $\lim _{x_{j} \rightarrow \underline{d}_{j}} t_{j}^{\prime}\left(x_{j}\right) \geq$ 0 . Hence $\delta_{j}>0$ and $\delta_{i}>0$. Then let $\bar{x}>\underline{d}$ be such that $t_{j}^{\prime}\left(x_{j}\right)<\delta_{j}<\delta_{i}<t_{i}^{\prime}\left(x_{i}\right)$ for every $x_{i} \leq f_{i}(\bar{x})$ and $x_{j} \leq f_{j}(\bar{x})$. Thus, for such $x_{i}$ and $x_{j}, t_{i}\left(x_{i}\right)>\delta_{i}\left(x_{i}-\underline{d}_{i}\right)$ and $t_{j}\left(x_{j}\right)<\delta_{j}\left(x_{j}-\underline{d}_{j}\right)$. Since, for every $x \in(\underline{d}, \bar{x})$,

$$
\int_{f_{j}(x)}^{f_{j}(\bar{x})} \frac{d z}{t_{j}(z)}=\int_{f_{i}(x)}^{f_{i}(\bar{x})} \frac{d z}{t_{i}(z)}
$$

we have

$$
\int_{f_{j}(x)}^{f_{j}(\bar{x})} \frac{d z}{\delta_{j}\left(z-\underline{d}_{j}\right)}<\int_{f_{i}(x)}^{f_{i}(\bar{x})} \frac{d z}{\delta_{i}\left(z-\underline{d}_{i}\right)}
$$

Thus

$$
\left(\frac{f_{j}(\bar{x})-\underline{d}_{j}}{f_{j}(x)-\underline{d}_{j}}\right)^{1 / \delta_{j}}<\left(\frac{f_{i}(\bar{x})-\underline{d}_{i}}{f_{i}(x)-\underline{d}_{i}}\right)^{1 / \delta_{i}}
$$

Hence there exists a positive number $k$ such that

$$
f_{i}(x)-\underline{d}_{i}<k\left(f_{j}(x)-\underline{d}_{j}\right)^{\delta_{i} / \delta_{j}}
$$

Recall that both $f_{i}:(\underline{d}, \bar{d}) \rightarrow\left(\underline{d}_{i}, \bar{d}_{i}\right)$ and $f_{j}:(\underline{d}, \bar{d}) \rightarrow\left(\underline{d}_{j}, \bar{d}_{j}\right)$ are smooth, one-to-one, and onto, and have strictly positive derivatives. Hence there exists a $\varphi:\left(0, \bar{d}_{j}-\underline{d}_{j}\right) \rightarrow\left(0, \bar{d}_{i}-\underline{d}_{i}\right)$ that is smooth, one-to-one, and onto, has strictly positive derivatives, and satisfies $f_{i}(x)-\underline{d}_{i}=$ $\varphi\left(f_{j}(x)-\underline{d}_{j}\right)$. Thus, also by inequality $(21), 0<\varphi(z)<k z^{\delta_{i} / \delta_{j}}$ for every $z \in\left(0, \bar{d}_{j}-\underline{d}_{j}\right)$. Hence, by $\delta_{j} / \delta_{i}>1, \varphi(z) / z \rightarrow 0$ and $\varphi^{\prime}(z) \rightarrow 0$ as $z \rightarrow 0$. If $z$ and $x$ satisfy $z=f_{i}(x)-\underline{d}_{i}$, then $z \rightarrow 0$ if and only if $x \rightarrow \underline{d}$. Hence $\left(f_{i}(x)-\underline{d}_{i}\right) /\left(f_{j}(x)-\underline{d}_{j}\right) \rightarrow 0$ as $x \rightarrow \underline{d}$. Moreover, since $\varphi^{\prime}(z)=f_{j}^{\prime}(x) / f_{i}^{\prime}(x), f_{j}^{\prime}(x) / f_{i}^{\prime}(x) \rightarrow 0$ as $x \rightarrow \underline{d}$.

We can now turn to the proof of Proposition 11.

Proof of Proposition 11 To show the first two parts, let $i \in \bar{I}$ and $j \notin \bar{I}$. Since $\limsup _{x \rightarrow \infty} f_{i}(x) / x \leq 1$,

$$
0 \leq \liminf _{x \rightarrow \infty} \frac{f_{j}(x)}{x} \leq \limsup _{x \rightarrow \infty} \frac{f_{j}(x)}{x} \leq \limsup _{x \rightarrow \infty} \frac{f_{j}(x)}{f_{i}(x)} \limsup _{x \rightarrow \infty} \frac{f_{i}(x)}{x} \leq \limsup _{x \rightarrow \infty} \frac{f_{j}(x)}{f_{i}(x)} .
$$

By Lemma 21, the far right hand side equals zero. Thus $f_{j}(x) / x \rightarrow 0$. Since this is true for every $j \notin \bar{I}$ and $\sum_{i=1}^{I} f_{i}(x) / x=1$, we must have $\sum_{i \in \bar{I}} f_{i}(x) / x \rightarrow 1$ as $x \rightarrow \infty$.

Also, since $0<f_{i}^{\prime}(x)<1$, we have $0<f_{j}^{\prime}(x)<\frac{f_{j}^{\prime}(x)}{f_{i}^{\prime}(x)}$ and, for such $i$ and $j$ as in the preceding paragraph, the far right hand side converges to zero as $x \rightarrow \infty$. Hence $f_{j}^{\prime}(x) \rightarrow 0$ 
as $x \rightarrow \infty$. We must have $\sum_{i \in \bar{I}} f_{i}^{\prime}(x) \rightarrow 1$ as $x \rightarrow \infty$. Since $\lim _{x_{j} \rightarrow \infty} t_{j}^{\prime}\left(x_{j}\right)<\lim _{x_{i} \rightarrow \infty} t_{i}^{\prime}\left(x_{i}\right) \leq \infty$ for every $i \in \bar{I}$ and $j \notin \bar{I}, t_{j}^{\prime}\left(f_{j}(x)\right) f_{j}^{\prime}(x) \rightarrow 0$ as $x \rightarrow \infty$ for every $j \notin \bar{I}$. Thus, by Lemma 1 and $0<\sum_{i \in \bar{I}} f_{i}^{\prime}(x) \leq 1$, we have

$$
\begin{aligned}
\limsup \sup _{x \rightarrow \infty} t^{\prime}(x) & =\lim \sup _{x \rightarrow \infty} \sum_{i=1}^{I} t_{i}^{\prime}\left(f_{i}(x)\right) f_{i}^{\prime}(x)=\lim \sup _{x \rightarrow \infty} \sum_{i \in \bar{I}} t_{i}^{\prime}\left(f_{i}(x)\right) f_{i}^{\prime}(x) \\
& \leq \lim \sup _{x \rightarrow \infty} \max _{i \in \bar{I}} t_{i}^{\prime}\left(f_{i}(x)\right) \leq \max _{i \in\{1, \ldots, I\}} \lim _{x_{i} \rightarrow \infty} t_{i}^{\prime}\left(x_{i}\right) .
\end{aligned}
$$

The other inequality,

$$
\max _{i \in\{1, \ldots, I\}} \lim _{x_{i} \rightarrow \infty} t_{i}^{\prime}\left(x_{i}\right) \leq \liminf _{x \rightarrow \infty} t^{\prime}(x),
$$

can be shown analogously. This proves the first two parts of this proposition.

To prove part 3 , let $i \in \underline{I}$ and $j \notin \underline{I}$. Since $0<\frac{f_{i}(x)-\underline{d}_{i}}{x-\underline{d}}<1$ for every $x$,

$0 \leq \liminf _{x \rightarrow \underline{d}} \frac{f_{j}(x)-\underline{d}_{j}}{x-\underline{d}} \leq \limsup _{x \rightarrow \underline{d}} \frac{f_{j}(x)-\underline{d}_{j}}{x-\underline{d}} \leq \limsup _{x \rightarrow \underline{d}} \frac{f_{j}(x)-\underline{d}_{j}}{f_{i}(x)-\underline{d}_{i}} \limsup _{x \rightarrow \underline{d}} \frac{f_{i}(x)-\underline{d}_{i}}{x-\underline{d}} \leq \limsup _{x \rightarrow \underline{d}} \frac{f_{j}(x)-\underline{d}_{j}}{f_{i}(x)-\underline{d}_{i}}$.

By Lemma 22, the far right hand side equals zero. Hence $\frac{\sum_{i \in \underline{I}}\left(f_{i}(x)-\underline{d}_{i}\right)}{x-\underline{d}} \rightarrow 1$ as $x \rightarrow \underline{d}$.

Part 4 can be shown in the same manner as for part 2 .

\section{References}

[1] Yacine Aït-Sahalia and Andrew W. Lo, 2000, Nonparametric risk management and implied risk aversion, Journal of Econometrics, Vol. 94, pp. 9-51.

[2] Robert B. Barsky, F. Thomas Juster, Miles S. Kimball, Matthew D. Shapiro, 1997, Preference parameters and behavioral heterogeneity: An experimental approach in the health and retirement study, Quarterly Journal of Economics, Vol. 112, pp. 537-579.

[3] Simon Benninga and Joram Mayshar, 2000, Heterogeneity and option pricing, Review of Derivatives Research, Vol. 4, pp. 7-27.

[4] Olivier J. Blanchard, 1993, Movements in the equity premium, Brookings Papers on Economic Activity, Macroeconomics, Vol. 2, pp. 75-118.

[5] Karl Borch, 1962, Equilibrium in a reinsurance market, Econometrica, Vol. 30, No. 3, pp. $424-444$.

[6] Michael J. Brennan and R. Solanki, 1981, Optimal portfolio insurance, Journal of Financial and Quantitative Analysis, Vol. 16, No. 3, pp. 279-300. 
[7] Laurent Calvet, Jean-Michel Grandmont, and Isabelle Lemaire, 1999, Aggregation of heterogeneous beliefs and asset pricing in complete financial markets, mimeo.

[8] John Y. Campbell and John H. Cochrane, 1999, By force of habit: A consumption-based explanation of aggregate stock market behavior, Journal of Political Economy, Vol. 107, pp. 205-251.

[9] Yeung Lewis Chan and Leonid Kogan, 2002, Catching up with the Joneses: Heterogeneous preferences and the dynamics of asset prices, Journal of Political Economy, Vol. 110, pp. 1255-1285.

[10] Bernard Dumas, 1989, Two-person dynamic equilibrium in the capital market, Review of Financial Studies, Vol. 2, pp. 157-188.

[11] Günter Franke, Richard C. Stapleton, Marti G. Subrahmanyam, 1999, When are options overpriced? The Black-Scholes model and alternative characterisations of the pricing kernel, European Finance Review, Vol. 3, pp. 79-102.

[12] Christian Gollier, 2001a, Economics of Time and Risk, MIT Press, Cambridge, Mass.

[13] Christian Gollier, 2001b, Wealth inequality and asset pricing, Review of Economic Studies, Vol. 68, pp. 181-203.

[14] Christian Gollier, 2005, Whom should we believe? Collective risk-taking decisions with heterogeneous beliefs, mimeo, University of Toulouse (LERNA and IDEI).

[15] Christian Gollier and Richard J. Zeckhauser, 2002, Horizon length and portfolio risk, Journal of Risk and Uncertainty, Vol. 24:3, pp. 195-212.

[16] Chiaki Hara, 2006, Heterogeneous risk attitudes in a continuous-time model, The Japanese Economic Review, Vol. 57, No. 3, pp. 377-405.

[17] Chiaki Hara and Christoph Kuzmics, 2005, Representative consumer's risk aversion and efficient risk-sharing rules, mimeo.

[18] Chi-Fu Huang and Richard Litzenberger, 1988, Foundations of Financial Economics, North-Holland, Amsterdam.

[19] Xiaoping (James) Huang, 2000a, Demand for and Pricing of Options, Ph.D. Thesis, Department of Accounting and Finance, Strathclyde University. 
[20] James Huang, 2000b, Who buys options from whom? The role of options in an economy with heterogeneous preferences and beliefs, Lancaster University Management School Working Paper 2000/023 (revised on May 2002).

[21] James Huang, 2002, Existence of an optimal portfolio for every investor in an ArrowDebreu economy, Lancaster University Management School Working Paper 2002/012.

[22] James Huang, 2003, Impact of divergent consumer confidence on option prices, Review of Derivatives Research, Vol. 6, pp. 165-177.

[23] Ravi Jagannathan, Ellen R. McGrattan, and Anna Scherbina, 2000, The declining US equity premium, Federal Reserve Bank of Minneapolis Quarterly Review, Vol. 24, 4, pp. $3-19$.

[24] David M. Kreps, 1990, A Course in Microeconomic Theory, Princeton University Press, Princeton.

[25] Takashi Kurosaki, 2001, Consumption smoothing and the structure of risk and time preferences: theory and evidence from village India, Hitotsubashi Journal of Economics, Vol. 42, pp. 103-117.

[26] Hayne E. Leland, 1980, Who should buy portfolio insurance, Journal of Finance, Vol. 35, No. 2, pp. 581-594.

[27] Stephen LeRoy, and Jan Werner, 2001, Principles of Financial Economics, Cambridge University Press, Cambridge.

[28] Michael Magill and Martine Quinzii, 1996, Incomplete Markets, MIT Press, Cambridge, Mass.

[29] Rajnish Mehra, and Edward C. Prescott, 1985, The Equity premium: A puzzle, Journal of Monetary Economics, Vol. 15, No. 2, pp. 145-61.

[30] Jiang Wang, 1996, The term structure of interest rates in a pure exchange economy with heterogeneous investors, Journal of Financial Economics, Vol. 41, No. 1, pp. 75-110.

[31] Philippe Weil, 1992, Equilibrium asset prices with undiversifiable labor income risk", Journal of Economic Dynamics and Control, Vol. 16, No. 3-4, pp. 769-790. 
[32] Robert Wilson, 1968, The theory of syndicates, Econometrica, Vol. 36, No. 1, pp. 119132. 